\title{
Sobrevivencia, metamorfosis y crecimiento de larvas del camarón Penaeus californiensis (Decapoda: Peneidae) alimentadas con diferentes microalgas
}

\author{
José Naranjo ${ }^{1}$, Marco A. Porchas ${ }^{1}$, Miguel Robles ${ }^{2}$, Francisco J. Magallón ${ }^{2}$, José Valdez ${ }^{3}$ y \\ Humberto Villarreal ${ }^{2}$
}

1 Centro de Investigaciones Biológicas del Noroeste S.C., Unidad Guaymas. Apdo. Postal 349, Guaymas, Sonora México. Fax (622) 1-22-38. Correo electrónico: jnaranjo@ cibnor.mx

2 Centro de Investigaciones Biológicas del Noroeste, S.C. 128, La Paz, B.C.S. Apdo. Postal 23000, México.

3 Instituto de Acuacultura del Estado de Sonora, Hidalgo 581. Col. Centenario. Hermosillo, Sonora. Apdo. Postal 83000. México.

Recibido 17-XI-1998. Corregido 1-VII-1999. Aceptado 5-VII-1999.

\begin{abstract}
The effect of three microalgae, Chaetoceros gracilis, Isochrysis galbana, and Dunalliella sp. on the larval development of yellowleg shrimp Penaeus californiensis, was evaluated in the Oyster Production Center of Bahia Kino, Sonora, México. The effects of each microalgae (1) individually, (2) combined and (3) mixed with Artemia nauplii (from zoea III stage), were considered. Twenty four experimental units (three 16.01 plastic bottles per treatment) were used. During the experiment temperature was maintained around $25^{\circ} \mathrm{C}$, and salinity around $35 \mathrm{ppt}$. The stocking density of nauplii was 50/1. The best survival was obtained with $C$. gracilis (55\%), followed by the combination of $C$. gracilis and Dunalliella sp (48\%). The worst survival was obtained in the treatment with Dunalliella sp. An evident delay in the metamorphosis times was observed in treatments without $C$. gracilis. Significant differences $(\mathrm{p}<0.05)$ in growth from zoea II stage were recorded in those treatments.
\end{abstract}

Key words: Shrimp, Penaeus californiensis, larval culture, nutrition, microalgae.

En los últimos años la camaronicultura ha mostrado un desarollo importante a nivel mundial; en 1995 alcanzó las 712000 toneladas métricas de camarón cultivado (Rosenberry 1995). En México el cultivo de camarón es una industria relativamente nueva, se han establecido varias de las granjas en los últimos diez años, especialmente en el noroeste del país (Ochoa 1994). Un obstáculo para su desarrollo en el noroeste de México es el régimen climático. Es una región semiárida, templada con temperaturas menores a $20^{\circ} \mathrm{C}$ en las costas, durante cuatro a cinco meses del año. Esto res- tringe el cultivo a un periodo de siete a ocho meses (marzo a octubre). Esto es una desventaja económica con respecto a otras regiones. Penaeus vannamei y Penaeus stylirostris son las principales especies de cultivo.

El camarón café P. californiensis es nativo de las costas del Golfo de California, puede crecer a bajas temperaturas por lo que es un buen candidato para el cultivo en el noroeste de México durante el invierno (Magallón et al. 1994).

La mayoría de los estudios sobre esta especie se relacionan con su distribución y pesquería (Olson-Ocampo 1982, Villavicencio 
1976, Luna-Serrano 1987), biología general (Olguín 1976), y la comparación entre juveniles de laboratorio y juveniles silvestres (Casillas et al. 1991). Hay pocas referencias sobre su fisiología, reproducción o larvicultivo. García (1994) registró el efecto de la temperatura en su desarrollo larvario; Porchas (1996) registró el efecto de la salinidad en el desarrollo larvario; Kitani y Alvarado (1981) estudiaron su desrrollo en laboratorio y Moore et al. (1974) realizaron un estudio sobre la maduración en cautiverio. Es necesario generar información complementaria relacionada con su biología y ecología.

Las dietas para larvas de peneidos son diversas y lejanas de ser óptimas. Dos preguntas de contínua importancia son: cúal es la mejor fuente de alimento algal y en que estadio de desarrollo podría ser introducido alimento animal en las dietas larvales para peneidos.

Varias especies de microalgas, como los fitoflagelados Isochrysis sp. y Tetraselmis sp. han sido usados para alimentar larvas de peneidos (Hudinaga 1942, Bardach et al. 1972, Kuban et al. 1983, 1985). Diatomeas como Skeletonema sp., Thalassiosira sp. y Chaetoceros sp. han sido tambien usadas exitosamente (Hudinaga 1942, Cook y Murphy 1966, Emerson 1980, Kuban et al. 1983).

Este estudio evalúa el efecto de varias microalgas en la sobrevivencia, crecimiento y velocidad de metamorfosis durante el cultivo larvario de camarón café $P$. californiensis, para contribuir al desarrollo de las técnicas de producción de postlarvas de esta especie, en condiciones de laboratorio.

\section{MATERIALES Y MÉTODOS}

Los organismos experimentales fueron obtenidos de un desove múltiple en laboratorio a partir de 4 hembras maduras capturadas en Bahía de Kino, Sonora, México. Se usaron 800 nauplios (NV) para ser sembrados en cada unidad experimental, a una densidad de 50 nauplios/l, en 24 unidades experimentales, las cuales consistieron en garrafones plásticos de 181 invertidos y desprovistos del fondo.

El sistema experimental consistió de una estructura de madera con dos niveles, para soportar las 24 unidades experimentales, localizado en un cuarto con temperatura variable $\left(20-25^{\circ} \mathrm{C}\right)$. Cada unidad fue provista de agua marina filtrada a una micra y aeración moderada constante mediante un compresor de $3 \mathrm{HP} \mathrm{a}$ través de difusores de sílica colocados en el fondo de cada tanque. La salinidad del agua de las unidades fue de 35 ppmil y la temperatura se mantuvo a $25 \pm 0.5^{\circ} \mathrm{C}$ mediante calentadores sumergibles de 50 watts. Se proporcionó iluminación artificial mediante tubos fluorescentes de 40 watts. Se realizó un recambio diario de $20 \%$ del agua a partir del estadio de zoea II y gradualmente se incrementó hasta $100 \%$ en el estadio de mysis III.

El cultivo de microalgas se efectuó de una manera secuencial; se usó el medio f/2 de Guillard (1975) como medio de cultivo. Se emplearon matraces de $200 \mathrm{ml}$ seguidos de inoculaciones en matraces de dos litros, garrafones de vidrio de $18 \mathrm{l}$; cada fase de escalamiento duró dos días de crecimiento, de la fase de garrafones se alimentaron las unidades de cultivo larvario de $P$. californiensis.

Las microalgas utilizadas para alimentar a las larvas fueron la diatomea Chaetoceros gracilis y los fitoflagelados Isochrysis galbana y Dunaliella sp. solas o combinadas; se establecieron ocho tratamientos con tres réplicas en las 24 unidades experimentales (Cuadro 1).

Las concentraciones de microalgas usadas en este trabajo se determinaron con base en lo recomendado en la literatura (Kuban et al 1983, 1985, Naranjo et al 1994, Villarreal y Naranjo 1994, Porchas 1996) para diferentes especies de peneidos incluyendo a $P$. californiensis.

Para evaluar el efecto de las diferentes microalgas en cada subestadio, se registró el desarrollo larvario, la sobrevivencia, la velocidad de metamorfosis y el crecimiento. Las diferencias en estos parámetros fueron definidas por análisis de varianza y prueba de rangos múltiple de Tukey. 


\section{CUADRO 1}

Tratamientos experimentales con diferentes microalgas para larvas de Penaeus californiensis.

Experimental treatments, with diferent microalgae for larvae of Penaeus californiensis.

$\begin{array}{cccc}\text { Tratamientos (@) } & \begin{array}{c}\text { Chaetoceros gracilis } \\ (\mathrm{C})^{*}\end{array} & \begin{array}{c}\text { Isochrysis galbana } \\ (\mathrm{I})^{*}\end{array} & \begin{array}{c}\text { Dunaliella sp. } \\ (\mathrm{D})^{*}\end{array} \\ \text { 1. C } & 100 & 0 & 0 \\ \text { 2. I } & 0 & 100 & 0 \\ \text { 3. D } & 0 & 0 & 100 \\ \text { 4. C,D } & 80 & 0 & 20 \\ \text { 5. I,D } & 0 & 80 & 20 \\ \text { 6. C, I } & 20 & 80 & 0 \\ \text { 7. C,I,D } & 70 & 20 & 10 \\ \text { 8. I^ } & 0 & 100 & 0\end{array}$

* X $1000(\mathrm{Cel} / \mathrm{ml})$, @ Artemia: $0.25 \mathrm{~N} / \mathrm{ml}$ a partir de ZIII, ^No se agregó Artemia durante la prueba.

\section{RESULTADOS}

En la fig. 1 se muestran las sobrevivencias obtenidas al estadio de postlarva (PL1) para los distintos tratamientos; fueron evidentes diferencias estadísticamente significativas $(\mathrm{p}<$ $0.05)$ entre tratamientos.

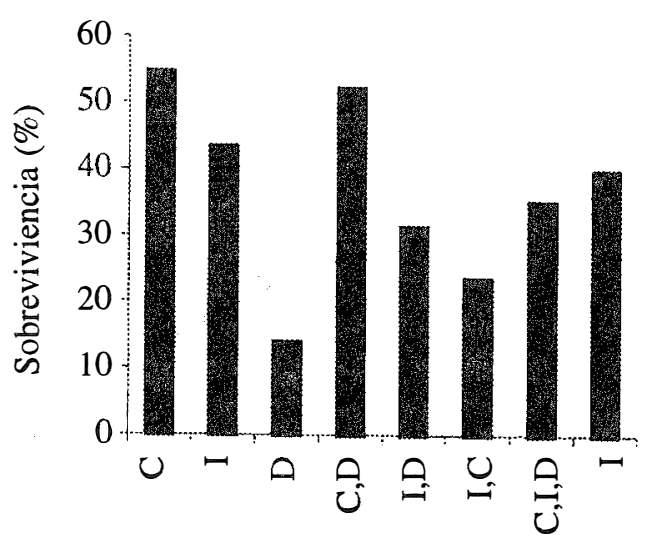

Tratamientos

Fig. 1. Sobrevivencia al estado de postlarva 1 de Penaeus californiensis alimentado con diferentes microalgas durante su desarrollo larvario.

Fig. 1. Survival to postlarva of Penaeus californiensis fed with different microalgae during larval development.
La sobrevivencia más alta fue obtenida en el tratamiento de Chaetoceros gracilis (55\%); siguiendole la combinación $C$. gracilis más Dunaliella sp. (52 \%). Donde se utilizó Isochrysis galbana más Artemia sp. e I. galbana sin Artemia sp. las sobrevivencias obtenidas fueron 43 y $40 \%$ respectivamente. Donde Artemia sp. no fue adicionada la condición de la larva fue muy débil. La sobrevievencia más baja fue obtenida donde se utilizó Dunaliella sp. más Artemia sp.

En cuanto al tamaño de las larvas, fueron evidentes diferencias significativas $(\mathrm{p}<0.05)$ a partir del estadio de zoea II (ZII), ver Fig. 2. El tamaño más alto a PL1 se obtuvo en los tratamientos donde se utilizó I. galbana más Dunaliella sp. e I. galbana más C. gracilis $(5.32$ y $5.31 \mathrm{~mm}$, respectivamente).

Fue evidente un retraso para la velocidad de metamorfosis de las larvas en los tratamientos en los que $C$. gracilis no fue utilizada. Donde se utilizó esta microalga las larvas alcanzaron el estadio de PL1 en 14 días, excepto en el tratamiento donde se utilizó $C$. gracilis, I. galbana y Dunaliella sp. (15 días) y Dunaliella sp. Los tratamientos con I. galbana con y sin Artemia sp. tardaron 17 días en llegar al estadio de PL1, ver fig. 3. 


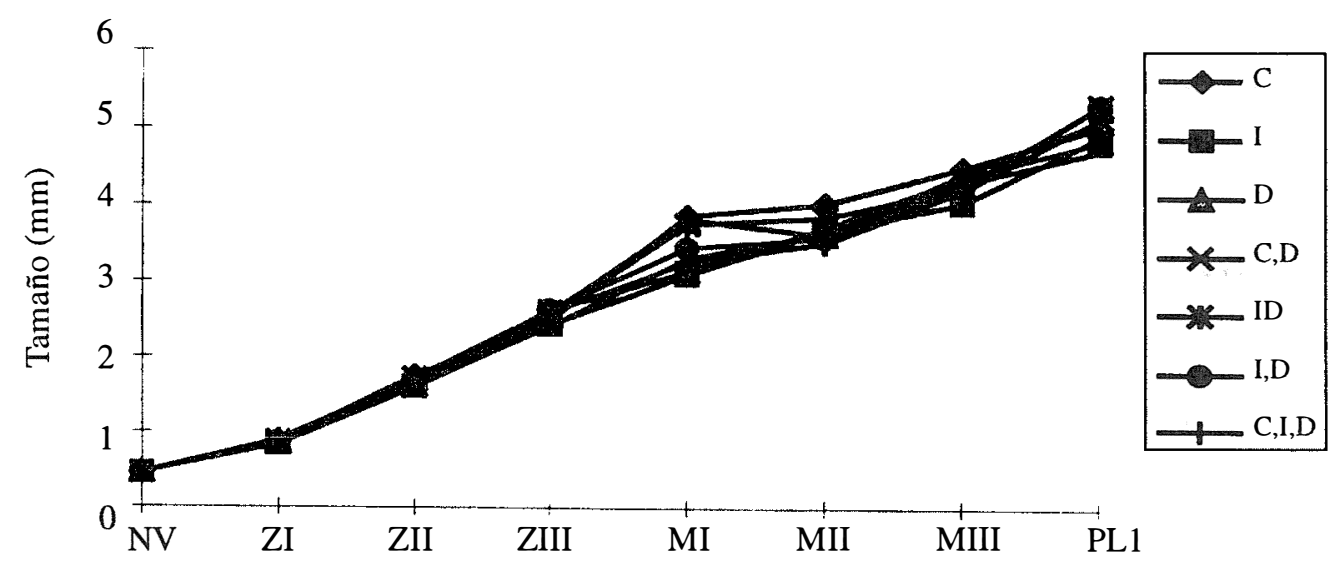

Subestarios larvarios

Fig. 2. Tamaño de subestadios larvarios de Penaeus californienses alimentados con diferentes microalgas.

Fig. 2. Size of larval substages of Penaeus californiensis fed with different microalgae.

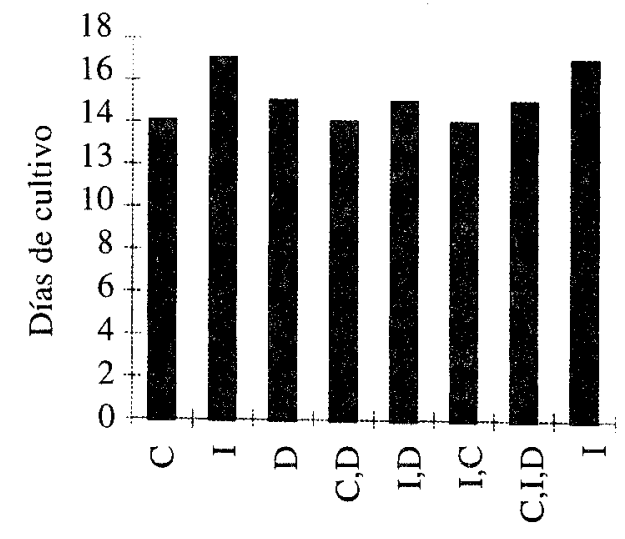

Tratamientos

Fig. 3. Metamorfosis de larvas de Penaeus californienses alimentados con diferentes microalgas.

Fig. 3. Metamorphosis of lavae Penaeus californiensis fed with different microalgae.

\section{DISCUSIÓN}

Con base en los resultados de sobrevivencia obtenidos en el presente trabajo, $C$. gracilis es recomendada como una buena fuente nutricional para larvas de $P$. californiensis. En este estudio sólo se utilizó esta diatomea; en los tratamientos donde $C$. gracilis fue adicio- nada ya sea sola o combinada se obtuvieron las mejores sobrevivencias. En general se han reportado buenos resultados en cuanto a sobrevivencia y metamorfosis, cuando se hace uso de diatomeas como fuente de alimento para larvas de camarón. Cuando Kuban et al. (1985), utilizaron fitoflagelados (Isochrysis sp. y Tetraselmis chuii) los intervalos de sobrevivencia no fueron mejorados para $P$. aztecus, $P$. vannamei, $P$. setiferus y $P$. stylirostris. Cook y Murphy (1966), observaron un incremento en la sobrevivencia de P. aztecus cuando las larvas se alimentaron con Skeletonema sp. Sin embargo, Isochrysis sp., ha sido utilizada exitosamente en pruebas anteriores para $P$. californiensis (García 1994, Porchas 1996), obteniendo mejores sobrevivencias que en el presente trabajo.

La variabilidad en resultados obtenidos con diferentes microalgas y distintas especies puede ser atribuido a las variaciones estacionales en la calidad de la microalga, y a la fase de cultivo en que se utilice. Guillard (1975) mencionó que la mejor composición bioquímica o nutricional de una microalga se presenta cuando ésta alcanza la fase exponencial de cultivo, por lo que lo anterior debe tambien ser evaluado. Encinas $e t$ al. (1992), reportaron variabilidad estacional para 
dos de las microalgas utilizadas en este trabajo (C. gracilis e I. galbana), registrando una tendencia significativa a presentar mayor producción de biomasa durante el invierno, de igual manera la composición bioquímica se eleva en dicha estación sobre todo para el caso de carbohidratos, seguido de lípidos y en menor medida para proteínas .

Los tamaños de los subestadios larvarios en el presente trabajo son similares a los reportados por Kitani y Alvarado (1981) para esta especie, y son menores a los registrados por Porchas et al. (1996), para los estadios de zoea III a postlarva 1 (PL1).

Los resultados sugieren que las diatomeas son mejor fuente nutricional que los fitoflagelados en cuanto a velocidad de metamorfosis se refiere, lo que podría deberse a una deficiencia nutricional en donde se usaron diatomeas. Kuban et al. $(1983,1985)$ observaron resultados similares para $P$. vannamei y $P$. stylirostris. El completar en menor tiempo el desarrollo larvario representa ahorros significativos para los laboratorios de producción de postlarvas. Kuban et al. (1985) menciona que los requerimientos nutricionales entre larvas de peneidos pueden ser muy diferentes, lo cual se corrobora con resultados obtenidos usando las mismas microalgas como alimento para larvas de distintas especies de peneidos.

Los experimentos sobre alimentación larvaria basados en porcentajes de sobrevivencia ó metamorfosis, a menudo referidos como crecimiento, pueden no ser el mejor método para probar el valor nutricional de una dieta, si el crecimiento en términos de biomasa no es llevado a cabo (Kuban et al. 1985).

Es necesaria más investigación para definir los requerimientos nutricionales específicos para cada subestadio larvario, así como los régimenes de alimentación y la variabilidad estacional en la calidad de la microalga, se requieren criterios confiables para determinar la calidad de postlarvas producidas en laboratorio.

Para optimizar la producción de camarón en el noroeste de México, es necesario el desarrollo de técnicas de cultivo para especies bien adaptadas a las condiciones regionales, tales como el camarón café $P$. californiensis.

\section{RESUMEN}

Se realizó una prueba experimental en un Centro de Producción Ostrícola en Bahía de Kino, Sonora, con el fin de evaluar el efecto de diferentes microalgas en el desarrollo de larvas de camarón café Penaeus californiensis. Las microalgas evaluadas fueron la diatomea Chaetoceros gracilis y los fitoflagelados Isochrysis galbana y Dunalliella sp., solas o en combinación y adición de nauplios de Artemia sp. a partir del estadio de zoea III, excepto en un tratamiento donde no se adicionó Artemia sp. Se utilizaron 24 unidades experimentales (tres por tratamiento), consistentes en garrafones plásticos de 161 de capacidad, una densidad de siembra de 50 nauplios/l, una temperatura de $25^{\circ} \mathrm{C}$ controlada mediante calentadores sumergibles de 50 watts y una salinidad de 35 ppmil. C. gracilis produjo la mejor sobrevivencia (55\%), siguiendole la combinación de $C$. gracilis con Dunaliella sp. (48\%), la más baja sobrevivencia fue para el tratamiento donde se utilizó Dunaliella sp. fueron evidentes un retraso en la velocidad de metamorfosis para los tratamientos donde $C$. gracilis no fue utilizada y diferencias significativas $(p<0.05)$ en crecimiento a partir de zoea II. Los tamaños de los estadios larvarios en el presente trabajo son similares a los reportados en la literatura.

\section{REFERENCIAS}

Bardach, J.E., J.H. Ryther \& W.O. McLarney 1972. Aquaculture: The Farming and Husbandry of Freshwater and Marine Organisms. Wiley Interscience, Nueva York. 868 pp.

Casillas, H. R., G. Portillo \& F.J Magallón 1991. Differences in growth observed in small juvenile Penaeus californiensis between laboratory and coastal environments. World Aquaculture Conference \& Exposition. San Juan Puerto Rico. June 16th-20th. 1991.

Cook, H.L. \& M.A. Murphy. 1966. Rearing penaeid shrimp from eggs to postlarvae. Proc. Conf. Southeast Assoc. Game Comm. 19: 283-288.

Emerson, W.D. 1980. Ingestion, growth and development of Penaeus indicus larvae as a function of Thalassiosira weissfloggi cell concentration. Mar. Biol. 58: 65-73.

Encinas A. R. \& A.C. García. 1992. Composición bioquímica de dos especies de microalgas, durante invierno y primavera en un sistema de producción de 
larvas de ostión. Tesis de Licenciatura. Universidad de Sonora, México. 83 pp.

García, G. M. 1994. Influencia de la temperatura en el desarrollo larvario de camarón café Penaeus californiensis. Tesis de Licenciatura. Universidad Autónoma de Baja California Sur, México. 82 pp.

Guillard, R. R. 1975. Culture of phytoplankton for feeding marine invertebrates. pp. 29-60. In: W.L. Smith and M.H. Chanley (Editors), Culture of Marine Invertebrate Animals. Plenum, Nueva York

Hudinaga, M. 1942. Reproduction, development and rearing of Penaeus japonicus Bate. Jpn. J. Zool. 10: 305-393.

Kitani, H. \& J.N. Alvarado. 1981. The larval development of the pacific brown shrimp Penaeus californiensis reared in laboratory. Bull. Jap. Soc. Sci. Fish. 48: 375-389.

Kuban, F.D., J.S. Wilkenfeld \& A.L. Lawrence 1983. Survival and growth of Penaeus setiferus $\mathrm{L}$. and $\mathrm{Pe}$ naeus aztecus Ives larvae fed Artemia beginning at the protozoea two substage versus the mysis-one substage. J. World Maricult. Soc. 14: 38-48.

Kuban, F.D., A.L. Lawrence \& J.S. Wilkenfeld 1985. Survival, metamorphosis and growth of larvae from four penaeid species fed six food combinations. Aquaculture 47: 151-162.

Luna-Serrano, J.I. 1987. Distribución, abundancia y estructura poblacional de Penaeus californiensis Holmes 1990, en Bahía Todos Santos B.C.S. México. Tesis de Licenciatura. Universidad Autonoma de Baja California Sur, México. 64 pp.

Magallón, B.F., G. Portillo, R. Campos, M. A. Porchas, J. Naranjo \& A. Muhlia. 1994. Penaeus californiensis as a cold tolerant species in Baja California Sur, México. Book of Abstracts. Aquaculture' 94 : World Aquaculture Society. Nueva Orleans, p. 84.
Moore, D., R. Sherry \& F. Montañes 1974. Maturation of Penaeus californiensis in captivity. Proc. World Mariculture Society 5: 445-449.

Naranjo J., H. Villarreal., M.U. García \& M.A. Porchas 1994. Effect of temperature on metamorphosis, growth and survival of brown shrimp (Penaeus californiensis) larvae, with a note on its relationship to postlarval development. Book of Abstracts. Aquaculture'94: W.A.S. Nueva Orleans, pp. 93.

Ochoa, V. 1994. Situación nacional del cultivo de camarón. Camarón '94: Seminario Internacional de Camaronicultura en México. Mazatlan, Sinaloa, México.

Olguín, M. 1976. Contribución al estudio de la biología del camarón café Penaeus californiensis Holmes, 1990. Tesis de Bachiller. Instituto Nacional de la Pesca. México. 56 pp.

Olson-Ocampo, R. 1982. Los camarones litorales de bahía Todos Los Santos, B.C. México. Sistemática Distribución y Ecología (Crustacea, Decapoda, Natantia). Tesis de Licenciatura. Universidad Autonoma de Baja California Sur, México. 160 pp.

Porchas, C. M.A. 1996. Efecto de la salinidad en el desarrollo laravario del camarón café Penaeus californiensis (Holmes, 1900). Tesis de Licenciatura. Universisdad de Sonora. Departamento de Ciencias Químico-Biológicas. 64 p.

Rosenberry, B. 1995. World Shrimp Farming. Book Annual Report. Shrimp News International. pp. 68.

Villarreal H. \& J. Naranjo 1994. Survival, metamorphosis and growth of white shrimp (Penaeus vannamei) larvae fed nine food combinations. Book of Abstracts. Aquaculture'94: W.A.S. Nueva Orleans, pp. 110.

Villavicencio, C. 1976. Estudios de maduración sexual del camarón cafe $P$. californiensis y del camarón azul $P$. stylirostris. Mem. Symp. Dinámica poblacional de Camarones. Sic. Pesca, Instituto Nacional de la Pesca. México. Tomo II 427-446 p. 